\title{
MINIMUM EIGENVALUES FOR POSITIVE, ROCKLAND OPERATORS ${ }^{1}$
}

\author{
A. HULANICKI, J. W. JENKINS, AND J. LUDWIG
}

\begin{abstract}
Let $I$, be a positive, Rockland operator of homogeneous degree $\gamma$. The minimum eigenvalue of $d \pi(L)$ increases as the $\gamma$ th power of the homogeneous distance from the origin of the orbit corresponding to $\pi$.
\end{abstract}

Let $G$ be a connected, simply connected nilpotent Lie group with Lie algebra $\mathfrak{g}$. Let $A$ be a diagonalizable operator on $g$ with eigenvalues $1=\gamma_{1}<\cdots<\gamma_{p}$, such that for each $r>0$, the operator $\delta_{r}=r^{A}$ is an automorphism of $g$ and, hence, determines an automorphism, again denoted by $\delta_{r}$, of $G$. Let $|\cdot|$ denote a homogeneous gauge on $G$; i.e., $|\cdot|$ is a continuous, nonnegative function on $G$ with $|g|=0$ if, and only if, $g=e$, and satisfying $\left|\delta_{r} g\right|=r|g|$ for $r>0$. Let $\mathrm{g}^{*}$ be the dual of $\mathfrak{g}$, and let $\delta_{r}^{*}$ denote the adjoint of $\delta_{r}$ acting on $\mathrm{g}^{*}$. We define $|\cdot|$ on $\mathrm{g}$ by $|X|=|\exp X|$ and on $\mathrm{g}^{*}$ as follows: fix a basis $\left\{X_{1}, \ldots, X_{d}\right\}$ of $g$ consisting of eigenvectors of $A$, and let $\left\{X_{1}^{*}, \ldots, X_{d}^{*}\right\}$ be the dual basis of $\mathrm{g}^{*}$. For $\xi \in \mathrm{g}{ }^{*}$ we set $|\xi|=\left|\Sigma\left(\xi, X_{i}\right) X_{i}\right|$. One easily sees that $\left|\delta_{r} X\right|=r|X|$ and $\left|\delta_{r}^{*} \xi\right|=r|\xi|$ for $X \in \mathrm{g}, \xi \in \mathrm{g}^{*}$, and $r>0$.

Let $g \rightarrow \mathrm{Ad}^{*} g$ be the coadjoint representation of $G$ on $\mathrm{g}^{*}$, and for $\xi \in \mathrm{g}^{*}$ let $O(\xi)=\operatorname{Ad}^{*} G \cdot \xi$. Set

$$
|O(\xi)|=\inf \left\{\left|\xi^{\prime}\right| \mid \xi^{\prime} \in O(\xi)\right\} .
$$

By Kirillov theory [1] the equivalence classes of irreducible unitary representations of $G, \hat{G}$, can be identified with $\left\{O(\xi) \mid \xi \in \mathrm{g}^{*}\right\}$. Given $\xi \in \mathrm{g}^{*}$, we denote by $\pi_{\xi}$ the element of $\hat{G}$ corresponding to $O(\xi)$, and by $d \pi_{\xi}$ the resulting representation of $\mathscr{U}(\mathfrak{g})$, the universal enveloping algebra of $\mathfrak{g}$.

An element $L$ of $\mathscr{U}(g)$ is said to be homogeneous of degree $\gamma$ if $L\left(f \circ \delta_{r}\right)=$ $r^{\gamma}(L f) \circ \delta_{r}$ for all smooth functions of $f$. As in [2], a homogeneous operator $L$ is called a Rockland operator if $d \pi_{\xi}(L)$ is injective (on the space of smooth vectors) for each $\xi \neq 0$. $L$ is said to be positive if $(L f, f) \geqslant 0$ for all $f \in C_{c}^{\infty}(G)$. The proof by Nelson and Steinspring [6] that $d \pi(L)$ is essentially selfadjoint for any elliptic operator and any unitary representation $\pi$ uses, in fact, only the hypoellipticity of $L$. Thus, by the theorem of Helffer and Nourrigat [3] $d \pi_{\xi}(L)$ is essentially selfadjoint for any Rockland operator. Therefore, if $L$ is a positive, Rockland operator, both $L$ and $d \pi_{\xi}(L)$ are infinitesimal generators of contraction semigroups. In [2] Folland

Received by the editors October 31, 1983 and, in revised form May 22, 1984 and July 14, 1984. 1980 Mathematics Subject Classification. Primary 22E30, 43A55.

${ }^{1}$ This research was funded in part by National Science Foundation Grant MCS810078. 
and Stein show that the densities of the semigroup generated by $L,\left\{P_{t}\right\}_{t>0}$, are Schwartz functions on $G$. One can easily show that the infinitesimal generator of $\left\{\pi_{\xi}\left(P_{t}\right)\right\}_{t>0}$ is $d \pi_{\xi}(L)$. Since $\pi_{\xi}$, integrated to $L^{1}(G)$, maps into compact operators, $d \pi_{\xi}(L)$ has as eigenvalues a discrete subset $\sigma_{\xi}(L) \subset(0, \infty)$ for each $0 \neq \xi \in \mathfrak{g}^{*}$.

ThEOREM. Let $L$ be a positive, Rockland operator of degree $\gamma$. There is a $c>0$ such that, for all $\xi \in \mathrm{g}^{*}, \min \left\{\alpha \in \sigma_{\xi}(L)\right\} \geqslant c|O(\xi)| \mathfrak{g}^{\gamma}$.

Proof. Define $\delta_{r}^{*}$ on $g^{*}$ by $\left(\delta_{r}^{*} \xi, X\right)=\left(\xi, \delta_{r} X\right)$. Then

$$
d \pi_{\sigma_{r}^{*} \xi}(L) \cong d_{\pi_{\xi}}\left(L \circ \delta_{r}\right)=r^{\gamma} d \pi_{\xi}(L)
$$

Indeed, if $\alpha \in \operatorname{Aut}(G)$, we may also regard $\alpha$ as an automorphism of $g$ and similarly define $\alpha^{*} \in \operatorname{End}\left(\mathrm{g}^{*}\right)$. If $\pi \in \hat{G}$ we set $\pi^{\alpha}(x)=\pi(\alpha(x))$. One can easily verify from Kirillov theory that $\pi_{\xi}^{\alpha}=\pi_{\alpha^{*} \xi}$. This implies (i) for $\alpha=\delta_{r}$ and $L$ homogeneous of degree $\gamma$.

From (i) one easily shows

$$
\min \left\{\alpha \in \sigma_{\delta_{s}^{*} \xi}(L)\right\} \geq s^{\gamma} \min \left\{\alpha \in \sigma_{\xi}(L)\right\}
$$

by merely noting that $f$ is an eigenvector $d \pi_{\delta_{s}^{*} \xi}(L)$ if, and only if, $f \circ \delta_{1 / s}$ is an eigenvector for $d \pi_{\xi}\left(\delta_{s} L\right)=s^{\gamma} d \pi_{\xi}(L)$, and the corresponding eigenvalues differ by factor of $s^{\gamma}$.

We now set $B=\left\{\xi \in \mathrm{g}^{*}|1=| \xi|=| O(\xi) \mid\right\}$. It is clear that $B$ is compact and for each $\xi \in \mathrm{g}^{*}$ there is a $\xi^{\prime} \in B$ such that $O(\xi)=O\left(\delta_{s}^{*} \xi^{\prime}\right)$, where $s=|O(\xi)|$. Also, one has

$$
\inf \left\{\alpha \mid \alpha \in \sigma_{\xi}(L), \xi \in B\right\}>0 .
$$

To see this, use induction on $k$, where $B_{k}=\{\xi \in B \mid \operatorname{dim} O(\xi)=2 k\}$. The case $k=0$ is obvious, since for $\xi \in B_{0}, \pi_{\xi}$ is a character on $G$ and, thus, $\sigma_{\xi}(L)=$ $\left\{d \pi_{\xi}(L)\right\}$.

Assume $\inf \left\{\alpha \mid \alpha \in \sigma_{\xi}(L), \xi \in B_{l}, l<k\right\} \geqslant 0$ and suppose $\alpha_{n} \in \sigma_{\xi_{n}}(L), \xi_{n} \in B_{k}$, $\alpha_{n} \rightarrow 0$, and $\xi_{n} \rightarrow \xi_{0}$ in $\mathfrak{g}^{*}$. As in [1, p. 105] pick bases $\left\{X_{1}^{(n)}, \ldots, X_{d}^{(n)}\right\}$ of $g$ so that $\left\{X_{1}^{(n)}, \ldots, X_{d-k}^{(n)}\right\}$ is a basis for a polarization $\mathfrak{h}_{n}$ of $\xi_{n}$, so that $X_{i}=\operatorname{Lim} X_{i}^{(n)}$ exists for $1 \leqslant i \leqslant d$ and $\left\{X_{1}, \ldots, X_{d-k}\right\}$ is contained in a basis for a polarization of $\xi_{0}$. Define $\beta_{n}: \mathbf{R}^{d-k} \times \mathbf{R}^{k} \rightarrow G$ by

$$
\beta_{n}(\mathbf{t}, \mathbf{s})=\exp t_{1} X_{1}^{(n)} \cdots \exp s_{k} X_{d}^{(n)}
$$

where $\mathbf{t}=\left(t_{1}, \ldots, t_{d-k}\right)$ and $\mathbf{s}=\left(s_{1}, \ldots, s_{k}\right)$. Define $\tilde{\beta}_{n}: L^{2}\left(H_{n} \backslash G, \chi_{\xi_{n}}\right) \rightarrow L^{2}\left(\mathbf{R}^{k}\right)$ by

$$
\left(\tilde{\beta}_{n} f\right)(\mathbf{s})=f\left(\beta_{n}(0, \mathbf{s})\right)
$$

With the correct normalization of Lebesgue measure on $\mathbf{R}^{k}, \tilde{\beta}_{n}$ is an isometric isomorphism. Thus $\pi_{\xi_{n}} \simeq \tilde{\pi}_{\xi_{n}}=\tilde{\beta}_{n} \circ \pi_{\xi} \circ \tilde{\beta}_{n}^{-1}$. Also, one has that $\tilde{\pi}(g)=\operatorname{Lim} \tilde{\pi}_{\xi_{n}}(g)$ exists for each $g \in G$.

Let $\left\{P_{t}\right\}_{t>0}$ be the semigroup generated by $L$, and set

$$
R_{1}=\int_{0}^{\infty} e^{-t} P_{t} d t
$$


Then $R_{1}=(I+L)^{-1}$ (cf. [7]), and the assumptions imply that $\beta_{n}=1 /\left(1+\alpha_{n}\right) \in$ $\sigma_{\xi_{n}}\left(R_{1}\right)$, the spectrum of $\pi_{\xi_{n}}\left(R_{1}\right)$. Thus, $\sup \left\{\beta \in \sigma_{\xi_{n}}\left(R_{1}\right)\right\} \geqslant 1$. We will show that this is impossible.

In [5, Proposition 6.3], it is shown that, by passing to a subsequence if necessary, there are only finitely many orbits in $\mathrm{g}^{*}$, with dimension $2 k$, that are limit points in $\hat{G}$ of $\left\{O\left(\xi_{n}\right)\right\}$. Denote this set by $\mathscr{A}_{1}$. Let $\mathscr{A}_{2}$ be the lower-dimensional orbits that are limit points of $\left\{o\left(\xi_{n}\right)\right\}$, and let $\mathscr{A}=\mathscr{A}_{1} \cup \mathscr{A}_{2}$. Then, by [4],

$$
\lim _{n}\left\|\pi_{\xi_{n}}\left(R_{1}\right)\right\|=\sup _{o(\xi) \in \mathscr{A}}\left\|\pi_{\xi}\left(R_{1}\right)\right\| \text {. }
$$

Thus,

$$
\lim \sup _{n}\left\{\beta \in \sigma_{\xi_{n}}\left(R_{1}\right)\right\}=\max \left\{\sup _{\mathscr{A}_{1}}\left\|\pi_{\xi}\left(R_{1}\right)\right\|, \sup _{\mathscr{A}_{2}}\left\|\pi_{\xi}\left(R_{1}\right)\right\|\right\}<1,
$$

since $\mathscr{A}_{1}$ is finite and the orbits in $\mathscr{A}_{2}$ have dimension less than $2 k$.

\section{REFERENCES}

1. A. Kirillov, Unitary representations of nilpotent Lie groups, Uspehi Mat. Nauk 17 (1962), 57-110.

2. G. Folland and E. Stein, Hardy spaces on homogeneous groups, Math. Notes 28, Princeton Univ. Press, Princeton, N. J., 1982.

3. B. Helffer et J. Nourrigat, Caracterisation des opérateurs hypoelliptiques homogenes invariants a gauche sur un groupe de Lie gradué, Comm. Partial Differential Equations 4 (1979), 899-958.

4. I. Kaplansky, The structure of certain operator algebras, Trans. Amer. Math. Soc. 70 (1951), 219-255.

5. J. Ludwig, G. Rosenbaum and J. Samuel, The elements of bounded trace in the $C^{*}$-algebra of a nilpotent Lie group (preprint).

6. E. Nelson and W. F. Stinespring, Representation of elliptic operators in an enveloping algebra, Amer. J. Math. 81 (1959), 547-560.

7. K. Yosida, Functional analysis, Springer, New York, 1965.

Mathematical Institute, Polish Academy of Sciences, Wrockaw, Poland

Department of Mathematics, State University of New York, Albany, New York 12222

Fakultät fưr Mathematik, Universität Bielefeld, Bielefeld, BundesRepublik Deutschland 